\title{
Chitin, Chitinase Responses, and Invasive Fungal Infections
}

\author{
Karina Vega and Markus Kalkum \\ Department of Immunology and Irell \& Manella Graduate School of Biological Sciences, Beckman Research Institute, \\ City of Hope, 1500 East Duarte Road, Duarte, CA 91010, USA \\ Correspondence should be addressed to Markus Kalkum, mkalkum@coh.org
}

Received 16 July 2011; Revised 15 September 2011; Accepted 16 September 2011

Academic Editor: Arianna Tavanti

Copyright $\odot 2012$ K. Vega and M. Kalkum. This is an open access article distributed under the Creative Commons Attribution License, which permits unrestricted use, distribution, and reproduction in any medium, provided the original work is properly cited.

\begin{abstract}
The human immune system is capable of recognizing and degrading chitin, an important cell wall component of pathogenic fungi. In the context of host-immune responses to fungal infections, herein we review the particular contributions and interplay of fungus and chitin recognition, and chitin-degrading enzymes, known as chitinases. The mechanisms of host chitinase responses may have implications for diagnostic assays as well as novel therapeutic approaches for patients that are at risk of contracting fatal fungal infections.
\end{abstract}

\section{Introduction}

Recipients of solid organs and hematopoietic cell transplants, AIDS patients, and burn victims are usually immunosuppressed for extended periods of time. Their prolonged immunosuppressive state is associated with a high risk of contracting invasive fungal infections (IFIs) [1,2]. Most IFIs advance rapidly and are often not diagnosed early enough for antifungal drugs to function with full efficacy; therefore, the majority of these infections lead to death [1].

In contrast to immunosuppressed patients, immunocompetent individuals are protected from fungal infections by their functional innate immune system, which readily recognizes and eliminates fungal invaders. Recognition of fungal cellular features by the immune system appears to be a key component of the human antifungal defense [3]. For example, $\beta$-glucan on the fungal cell wall is recognized as a pathogen-associated molecular pattern (PAMP) by dectin- 1 and activates pro- and anti-inflammatory cytokines in a myeloid-differentiation-primary-responsegene-88-(MYD88-) dependent signaling pathway [3-6]. An important component of the fungal cell wall that has not been fully explored as a PAMP is chitin, a polymer of $N$ acetylglucosamine $[3,7]$. Chitin is one of the most abundant biopolymers, probably almost as abundant as cellulose $[8$, 9] and is found on fungal cell walls and exoskeletons of numerous organisms including parasitic worms (helminths) and arthropods. Although humans do not biosynthesize chitin, they do express chitin degrading enzymes, known as chitinases [10-12]. There are two known human chitinases that have chitinolytic activity, chitotriosidase (CHIT-1) and acidic mammalian chitinase (AMCase), as well as multiple noncatalytically active chitinases called chi-lectins [11-14]. The functions of CHIT-1 and AMCase are unknown, but they are thought to aid in the defense of chitincontaining pathogens. For instance, in guinea pigs, serum chitotriosidase levels increase in response to systemic fungal infection [15]. That chitinase levels can vary in response to fungal infections suggests the possibility of using host chitinase responses as a diagnostic. However, several other stimuli can also upregulate chitinase activity [16-19] and counterproductively, several polymorphisms in the CHIT-1 and $A M C a s e$ genes are known to decrease chitinase activity [20-24]. Thus, there are several challenges to be overcome if chitinase responses were to be used in the diagnosis of fungal infections. More recently, recombinant CHIT-1 was shown to have antifungal properties both in vitro and in vivo, suggesting the possibility of a gene therapy approach [25]. This paper will explore chitinase responses to fungal infections, current knowledge about the mechanism of chitin recognition by host-immune cells, and regulation of hostchitinase induction. 


\section{Invasive Fungal Infections (IFIs)}

Fungal infections have become a major disease concern over the last three decades, in particular for recipients of solid organs and hematopoietic stem cells, AIDS patients, and burn victims, all of whom are usually immunosuppressed for extended periods of time [26-28]. Their prolonged immunosuppressed status leads to an increased risk of contracting opportunistic IFIs. IFIs are also on the rise in intensive care settings, likely due to a growing use of procedures with invasive medical devices and long-term use of antibiotics [29]. In all cases, the most common etiological agents are Candida albicans and Aspergillus fumigatus [27, 29].

Humans are exposed to hundreds of fungal spores each day, usually without a negative effect on their health. In the lungs of patients that lack sufficient pulmonary immune defenses, A. fumigatus fungal spores are able to swell, germinate, and branch into fungal hyphae. The infection can then disseminate to other organs through the bloodstream $[26,30]$. Healthy individuals are able to eliminate fungal spores by mucociliary clearance, macrophages, and other primarily pulmonary defense mechanisms [26]. C. albicans, on the other hand, is a commensal organism residing in the gastrointestinal tract and oral, and vaginal mucosa of most healthy individuals, where it typically does not produce harmful side effects. However, Candida overgrowth can become symptomatic causing mucosal membrane infections, the most common being thrush and vaginal candidiasis [31-33]. Severe systemic Candida infections (Candidemia) and dissemination to internal organs can occur in immunocompromised patients [3133].

Current methods for detecting IFIs are based on clinical signs and microbial examination. For example, pulmonary fungal infections are typically examined via CT scan, followed up with bronchoalveolar lavage (BAL) and biopsy $[27,30]$. Systemic yeast infections such as candidiasis can be diagnosed by the blood culture [33]. However, current diagnostic methods usually do not detect fungal infections at early stages, and therefore, antifungal drug treatment is oftentimes inefficient or delayed. There are some serological tests that may be routinely performed assisting in the diagnosis of fungal infections via detection of fungal antigens in suspected patients [30]. For example, the galactomannan assay is sometimes used for the detection of Aspergillus in serum and BAL fluid. This assay works by detecting galactomannan released from the fungal pathogen by enzyme-linked immunosorbent assays [34, 35]. Elevated levels of galactomannan have been detected at early stages of fungal infections, however, the sensitivity and specificity of this assay has been criticized [36]. Moreover, the galactomannan assay is not useful for other fungal pathogens, including Candida [30, 37]. $\beta$-1,3-glucan serological detection assays are more widely used today because they can detect a wide range of fungi, including Aspergillus and Candida, but they do not detect zygo- or mucormycosis or cryptococcal disease [38]. The $\beta$-glucan assay works by detecting $\beta$-1,3-glucan, a major component of the fungal cell wall, circulating in the patient bloodstream $[39,40]$. The assay has had great promise for fungal detection, especially when used to confirm galactomannan positive results, however, problems with false-positive (and false-negative) results have been reported $[41,42]$. Despite the availability of such diagnostic tests, Garcia-Vidal et al. reported an increase in IFIs and lack of detection at an early stage, within 40 days after hematopoietic cell transplant in infected patients, demonstrating the ineffectiveness of present day diagnostic methods [27].

\section{Fungal Cell Wall Components and Pattern Recognition Receptors}

Generally, the innate immune system's response to PAMPs, which include glycosides, glycolipids, and carbohydrates, among others, involves pattern recognition receptors (PRRs) that are expressed by phagocytes. Pattern recognition then leads to a cascade of cellular signaling that activates phagocytes for defense $[3,26]$. The recognition of fungal cellular features, in particular fungal cell wall components, by the immune system of the host is an important element for mounting an antifungal defense response $[3,5,26,43]$. The fungal cell wall is composed of various mannoproteins, $\beta$ glucans as well as a thin, rigid layer of chitin (Figure 1). Many PRRs interact with fungal cell wall components. For example, mannoproteins with $O$-linked protein-carbohydrate conjugations are recognized by toll-like receptor (TLR)-4 [3, $44,45]$, while mannoproteins that are $\mathrm{N}$-linked can be recognized by dectin-2, mannose, and Fc $\gamma$ receptors [3, 45-47] (Figure 2). The galectin-3 receptor recognizes $\beta$ mannosides $[44,48-50]$. $\beta$-glucan is recognized as a PAMP by dectin-1 $[4,6]$ and when coated by phospholipomannan it is also recognized by both TLR-6 and TLR-2 [51-54]. Complement-coated $\beta$-glucan is recognized by complement receptor-3 $[55,56]$. Dectin-1 recognition of $\beta$-glucans results in an MYD88-dependent pathway activation $[3,5,44,55$, 57]. And finally, fungal CpG DNA is recognized by the intracellular receptor TLR-9 [58] (Figure 2). Recognition of fungal cell wall components by these PRRs generally leads to the nuclear factor kappa-lightchain enhancer of activated $\mathrm{B}$ cells $(\mathrm{NF}-\kappa \mathrm{B})$ signaling; this results in the activation of proinflammatory cytokines, such as tumor necrosis factor (TNF)- $\alpha$, or anti-inflammatory cytokines, such as interleukin (IL)-10 (Figure 2). Whether chitin in the fungal cell wall is recognized as a PAMP, and if a specific chitin receptor exists as a PRR, remains unknown; yet it is a very likely possibility $[3,7]$.

\section{Chitin as an Immune Modulator}

Intranasal or intraperitoneal chitin administration to mice caused an immunological preactivation effect, called priming, in alveolar macrophages and natural killer (NK) cells [65]. Shibata et al. examined the effects of chitin particle sizes on cellular responses, in particular macrophage activation and priming. Balb/c mouse splenocytes that were cocultured 


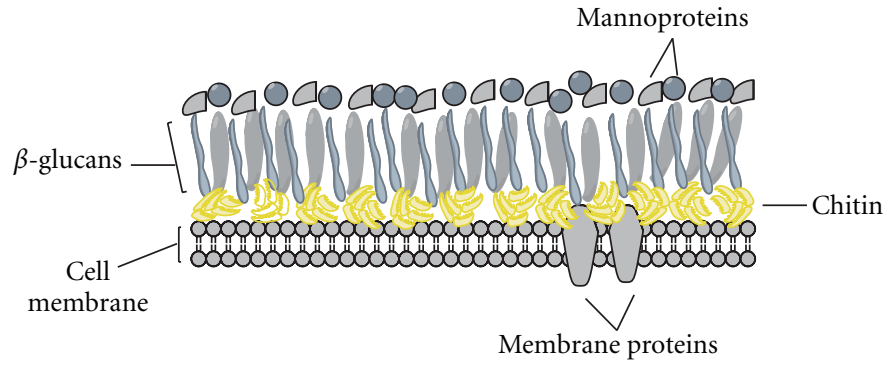

FIGURE 1: Fungal cell wall components. The fungal cell wall contains a cell membrane with various membrane proteins, a protective layer of chitin (yellow) as well as glucans (mostly beta), and mannoproteins on its surface. Different fungal cell walls contain different glucans. For example, the cell wall of A. fumigatus contains $\beta$-1,3- and $\beta$-1,4-glucan, and $\alpha$-1,3-glucan [30], while C. albicans contains $\beta$-1,3- and $\beta$-1,6-glucan [44].

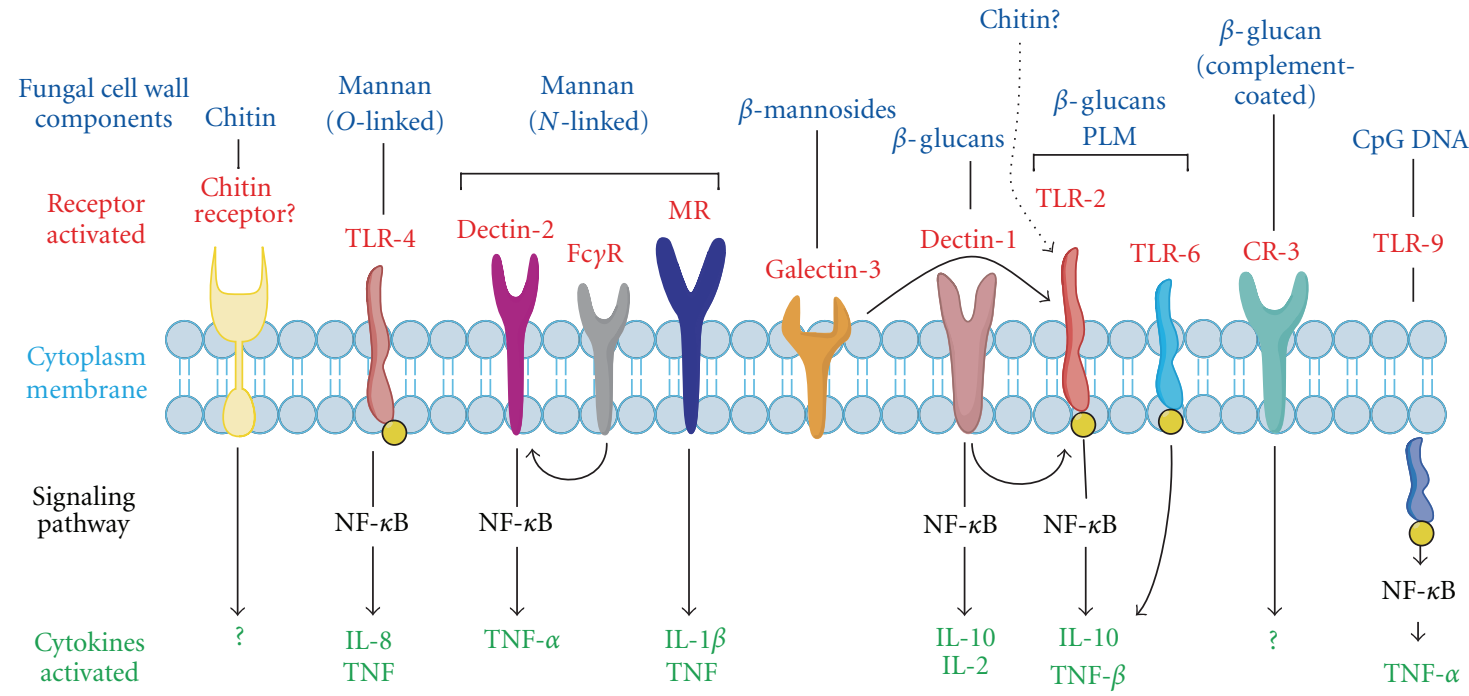

FIGURE 2: Fungal cell wall pathogen-associated molecular patterns (PAMPs) and their host-pattern recognition receptors (PRRs). Various fungal cell wall components are recognized by specific PRRs. Some PAMPs are recognized by multiple PRRs; for example, $N$-linked mannan is recognized by mannan receptor (MR), dectin-2, and Fc $\gamma \mathrm{R}[46,47]$. Phospholipomannan-(PLM-) coated $\beta$-glucans are recognized by both TLR-6 and TLR-2 $[53,54]$. Other receptors may involve the signaling pathway of another PRR. For example, galectin-3, which recognizes $\beta$-mannosides, signals through TLR-2 (represented by a curved arrow) $[47,49]$ dectin-1, when activated by $\beta$-glucans can signal to activate the nuclear factor kappa-lightchain enhancer of activated B cells (NF- $\kappa$ B) on its own or with the help of TLR-2 [4, 45]. Fc gamma receptor $(\mathrm{F} c \gamma \mathrm{R})$ may signal through dectin-2 when activated by $N$-linked mannan $[3,45,47,55]$. Recognition of these fungal cell wall components mediates fungal recognition and defense by the host. Recognition by host PRRs usually involves signaling through NF- $\kappa \mathrm{B}$ and activation of proinflammatory cytokines, such as TNF- $\alpha$, or in some instances, and anti-inflammatory cytokines such as interleukin (IL)-10 [45]. The possibility of an alternative chitin receptor exists, activation of which leads to the recruitment of IL-4 producing cells [45, 59]. However, chitin has been shown to function as a T helper (Th) 1 immune modulator, which stands in contrast an IL-4 associated Th2 response [60-64].

with chitin particles $(50-100 \mu \mathrm{m})$, produced, and secreted IL-12, TNF- $\alpha$, and IFN- $\gamma$ [60]. However, intravenous injection of phagocytosable small chitin particles $(1-10 \mu \mathrm{m})$ into C57 mice resulted in a macrophage priming that was dose dependent [61]. When utilizing a SCID mouse model instead of the C57 mice, the same chitin-macrophage priming effect was also found. Because SCID mice lack mature B and T cells, the authors concluded that neither $\mathrm{T}$ nor $\mathrm{B}$ lymphocytes were required for chitin-induced macrophage priming. An NK cell depletion experiment with anti-NK1.1 antibodies (antiCD161c) then demonstrated a requirement for NK cells and NK-secreted IFN- $\gamma$ in chitin-induced macrophage priming
[61]. However, as we describe below, chitin particles can also be used to activate macrophages and monocytes directly in cell-culture experiments.

It should be noted that chitin can also serve as an immunoadjuvant [65]. Orally administered chitin suppressed the production of $\mathrm{T}$ helper (Th) 2 cytokines and immunoglobulin (Ig)E in a ragweed allergy mouse model and induced IFN- $\gamma$ instead [62]. In addition, when used as an adjuvant, chitin produced Th1 responses comparable to other adjuvants, including heat-killed Mycobacterium bovis, Freud's complete adjuvant, and the Bacillus Calmette-Guérin vaccine [63]. Chitin produced effects similar to those of 
a Th1-promoting adjuvant in mouse models of ovalbumininduced asthma and allergic hypersensitivities induced by the house dust mite Dermatophagoides pteronyssinus and by the fungal pathogen A. fumigatus $[64,66]$. Chitin administration significantly reduced allergen-induced serum IgE levels and lung inflammation. Th1 cytokines IL-12, IFN- $\gamma$, and TNF- $\alpha$ were elevated, while IL- 4 levels were decreased in mice-administered chitin as compared to controls $[64,66]$. These and other studies strongly suggest that the immune system possesses a chitin recognition mechanism.

\section{Mammalian Chitinases}

Another immune response that may correlate with chitin recognition is the production of chitin-degrading enzymes, known as chitinases, by humans and other mammals. Chitinases belong to the glycosyl hydrolase 18 family, which is comprised of various proteins found in a wide range of organisms, including plants, bacteria, fungi, insects, protozoa, and mammals [13]. Six proteins with homology to chitinases have been identified in mammals. These include CHIT-1 and AMCase, which are the only two enzymatically active human chitinases able to hydrolyze chitin $[11,12$, 14]. The other four of these highly homologous members of the chitinase family contain amino acid substitutions at their active sites, rendering these proteins noncatalytic. These noncatalytic chitinases are referred to as chi-lectins or chitinase-like proteins, and include chitinase-3-like protein 1 (CHI3L1, also known as YKL-40, Hcgp39, or GP39), stabilin-interacting chitinase-like protein (SI-CLP), YKL-39 (chitinase 3-like protein 2), and oviductin [13].

CHIT-1 is highly expressed by activated macrophages and is used as a marker for macrophage stimulation, suggesting a possible role in innate immunity $[67,68]$. It was first discovered in the plasma of patients with Gaucher's disease; a disease characterized by the accumulation of lipidladen macrophages [68-70]. The use of a chitinase detection assay, which measures the presence of chitinase activity via cleavage of the fluorogenic substrate 4-methylumbelliferyl chitotriosidase, showed that CHIT-1 levels were elevated several hundred-fold in the plasma of patients with Gaucher's disease. Therefore, CHIT-1 is now being used as a biomarker for the diagnosis of Gaucher's disease [68, 69, 71]. These findings drew attention to the cloning and further characterization of CHIT-1 [72, 73] and the discovery of the other enzymatically active human chitinase, AMCase. The sequence of AMCase is highly homologous to that of CHIT1; however, AMCase is unique in that it functions strongest in acidic $\mathrm{pH}$ environments. Consistently, it was first found highly expressed in the stomach, intestinal tissue, and more recently is being studied as a biomarker for asthma and other hypersensitivities $[11,12,27,74]$.

Evolutionarily, chitinase production plays an important role in the life cycles of chitin-containing organisms such as fungi, insects and crustaceans, in which it is involved in either cell wall remodeling or molting. However, because mammals do not produce chitin, the physiological function of these chitinases and chi-lectins remains unclear, but various studies suggest that their function may lie in the digestion of chitin-containing foods and defense against chitin-containing pathogens and parasites $[11,13,75]$.

\section{Chitinases in Experimental Antifungal Therapy}

Chitinases have also been investigated for their potential use in antifungal therapy. Low concentrations of recombinant human CHIT-1 degraded the cell walls of Cryptococcus neoformans and visibly inhibited its growth in vitro [67]. Morphological changes, such as atypical blebs, hyphal tip bursting, and restrictions of hyphal growth, were also observed for Mucor rouxii and C. albicans in the presence of recombinant CHIT-1 [67]. In addition, recombinant human CHIT-1 induced a dose-dependent improvement in the survival of mice with $C$. albicans and A. fumigatus infections [67]. Recently, it was shown that the culture medium conditioned by Chinese hamster ovary cells that had been retrovirally transfected with the human CHIT-1 gene had antifungal activity [25]. These modified Chinese hamster ovary cells were then encapsulated in alginate microspheres and injected subcutaneously into BALB/c mice, where they continuously secreted active CHIT-1, and after infection with $C$. neoformans, mice harboring these cells had significantly lower fungal burden [25]. Therefore, the authors suggested that a continuous supply of active CHIT-1 should be explored in future gene therapies to prevent fungal infections.

\section{Mammalian Chitinase Responses to Inflammation and Fungal Infections}

Multiple stimuli, such as exposure to prolactin, interferon gamma (IFN- $\gamma$ ), lipopolysaccharides (LPS), and TNF- $\alpha$ can upregulate chitinase activity in human monocytes and macrophages, indicating a possible role for chitinase activity in inflammation [16-19]. Chitinase activity was reported to be upregulated as a result of various diseases, including candidiasis [76], Wuchereria bancrofti infections (filariasis) [21], and helminth infections $[77,78]$. AMCase activity is highly upregulated in individuals suffering from asthma, chronic rhinosinusitis, or allergic bronchopulmonary aspergillosis $[77,78]$. In addition, chitinase activity has been linked to fungal infections. In 1996, Overdijk et al. showed that, in guinea pigs, chitinase activity was induced after systemic infection with A. fumigatus $[15,79]$. Furthermore, mice with pulmonary $C$. neoformans exposure had increased AMCase chitinase activities in the airways [80]. Intraperitoneal injections of zymosan, a yeast-cell wall-derived product that contains beta-glucans and small quantities of chitin, was shown to increase serum chitinase activity of rats [81].

Although chitinase activity does not appear to be specific for fungal infections, as it is also upregulated in other diseases, there appears to be a correlation between chitinase activity and inflammation as well as with disease induced by chitin-containing pathogens. These findings suggest that mammalian chitinase responses to fungal infections 
and other parasitic infections may be triggered by the host response to a chitin-containing pathogen.

\section{Chitinase Induction and Regulation}

Little is known about how host chitinase activity is induced, but there is some indication that chitinase production and chitin recognition could be linked. Gorzelanny et al. used MALDI-TOF mass spectrometry to analyze the degradation of chitin by chitotriosidase and followed the stimulation of human monocyte/macrophage with a chitin hexamer [82]. These studies revealed that chitinases degrade chitin into smaller chitin-oligomers that in turn enhance the stimulation of macrophages, leading to more chitinase production [82]. However, the feedback mechanism of chitin recognition and chitinase secretion suggested by this study is still unclear and the signaling pathways involved are not fully understood.

Other chitin stimulation experiments revealed some aspects of the mechanism involved in the recognition of chitin and chitin-containing parasites by immune cells. Jumonji domain containing-3 (Jmjd3), a histone 3 Lys27 (H3K27) demethylase, along with Irf4 transcription factor, was determined to be essential for macrophage colony-stimulating factor (M-CSF)-bone-marrow-derived M2 macrophage polarization in response to Nippostrongylus brasiliensis helminth infection and chitin inoculation [59]. Another group found that mice exposed to $N$. brasiliensis helminth infection showed tissue invasion by macrophages and IL-4- and IL-13-producing immune cells as well as eosinophil recruitment [83]. Furthermore, transgenic mice that overexpressed AMCase in the lung, and were also exposed to $N$. brasiliensis, showed diminished infiltration of immune cells. The diminished infiltration of cells was likely due to $N$. brasiliensis chitin degradation and removal [83]. The same effect was observed when chitin alone was injected, and the effect was sustained, even in TLR-4deficient animals [83]. The latter effect is interesting, because TLR-4, which recognizes LPS and leads to activation of the innate immune system, was previously considered a possible chitin PRR candidate [44]. The observed recruitment of IL4 producing immune cells by chitin stands in stark contrast to the previously observed Th1 immune response induced by chitin when used as an adjuvant (see above). IL-4 is a typical Th2 response-inducing cytokine. It is possible though, that the IL-4 production by recruited immune cells is a secondary effect that requires other chemokines or other chemoattractants to be produced by primary chitin-sensing cells.

In contrast to TLR-4, TLR-2 and the IL17A receptor (IL17AR) may at least be partially involved in chitin recognition. Da Silva et al. reported that mouse macrophages stimulated with chitin particles had increased levels of IL-17 protein and IL-17AR mRNA, and the increase in IL-17 was mediated via the TLR-2 pathway. In vivo investigations demonstrated that chitin induces acute pulmonary inflammation in wild-type mice, but not in TLR-2 knockout mice [84]. Therefore, it is possible that TLR-2 and IL-17AR are somehow involved in the recognition of chitin. TLR-2 is known to recognize bacterial particles, LPS, and more interestingly, zymosan, which contains chitin (see above) [44].

Portions of the downstream signaling pathway that leads to chitinase expression have been analyzed. CHIT-1 mRNA expression in monocytes increases upon treatment with phorbol 12-myristate 13-acetate (PMA), which induces differentiation of monocytes into activated macrophages [85]. In addition, CHIT-1 gene activation is accompanied by the binding of phosphorylated CCAAT-enhancer-binding protein (C/EBP) $\beta$ and the transcription factor PU.1 to the promoter region of CHIT-1 (Figure 3) [85]. The upstream molecular signaling pathway leading to CHIT-1 gene activation and chitinase induction has not been determined; however, roles for some key proteins involved in chitinase regulation have been noted [16-19]. Chitinase gene expression and activity was induced by human-monocyte-derived macrophages after prolactin stimulation in both a dose- and time-dependent manner [17]. Because prolactin has similar structural properties as some proinflammatory cytokines, alternative stimulations were preformed with IFN- $\gamma$, TNF$\alpha$, and LPS, and, as a negative control, with IL-10, which has anti-inflammatory properties. Chitinase activity was elevated in human monocyte-derived macrophages after stimulation with IFN- $\gamma$, TNF- $\alpha$, and LPS and was significantly decreased after stimulation with IL-10 $[16,19]$. These findings may indicate the involvement of chitinase activity induction during inflammatory conditions. Prolactin stimulation of human monocyte-derived macrophages was also performed in the presence or absence of specific kinase inhibitors [18]. The phosphatidylinositol 3-kinase (PI3-K) inhibitors wortmannin and LY-294002 reduced chitinase activity, as did the protein tyrosine kinase inhibitor genistein, the mitogenactivated kinase (MAPK) p38 inhibitor SB203580, and the MAPK p44/42 inhibitor U0126. No effect was observed on prolactin-mediated chitinase induction when the controversial protein kinase $C$ inhibitor rottlerin was used, nor was an effect seen with PP2, a Src inhibitor, or AG490, a JAK2 inhibitor [18]. Accordingly, CHIT-1 induction can be mediated via a PI3-K/MAPK pathway (Figure 3).

\section{Polymorphisms in Chitinase Proteins}

The induction of chitinase activity as an immune response to various stimuli such TNF- $\alpha$, prolactin, and chitin, and in response to fungal infections suggests that chitinases are indeed involved in the host's immune response to a pathogenic fungal invader. However, multiple known polymorphisms can affect chitinase activity, the most prominent being a 24-bp duplication in the CHIT-1 gene. The CHIT-1 gene is composed of 12 exons and the protein is secreted as two isoforms. The major isoform has a molecular mass of $50 \mathrm{kDa}$, undergoes posttranslational modifications, including $\mathrm{O}$-linked glycosylation of the $\mathrm{C}$-terminal region (which contains the chitin-binding domain) and is alternatively spliced into the $39 \mathrm{kDa}$ minor isoform [20,68]. Sometimes, a 24-bp duplication occurs in exon 10 of CHIT-1 that causes a downstream cryptic $3^{\prime}$ splice site that generates mRNA with an in-frame deletion of 87 nucleotides (29 amino acids). 


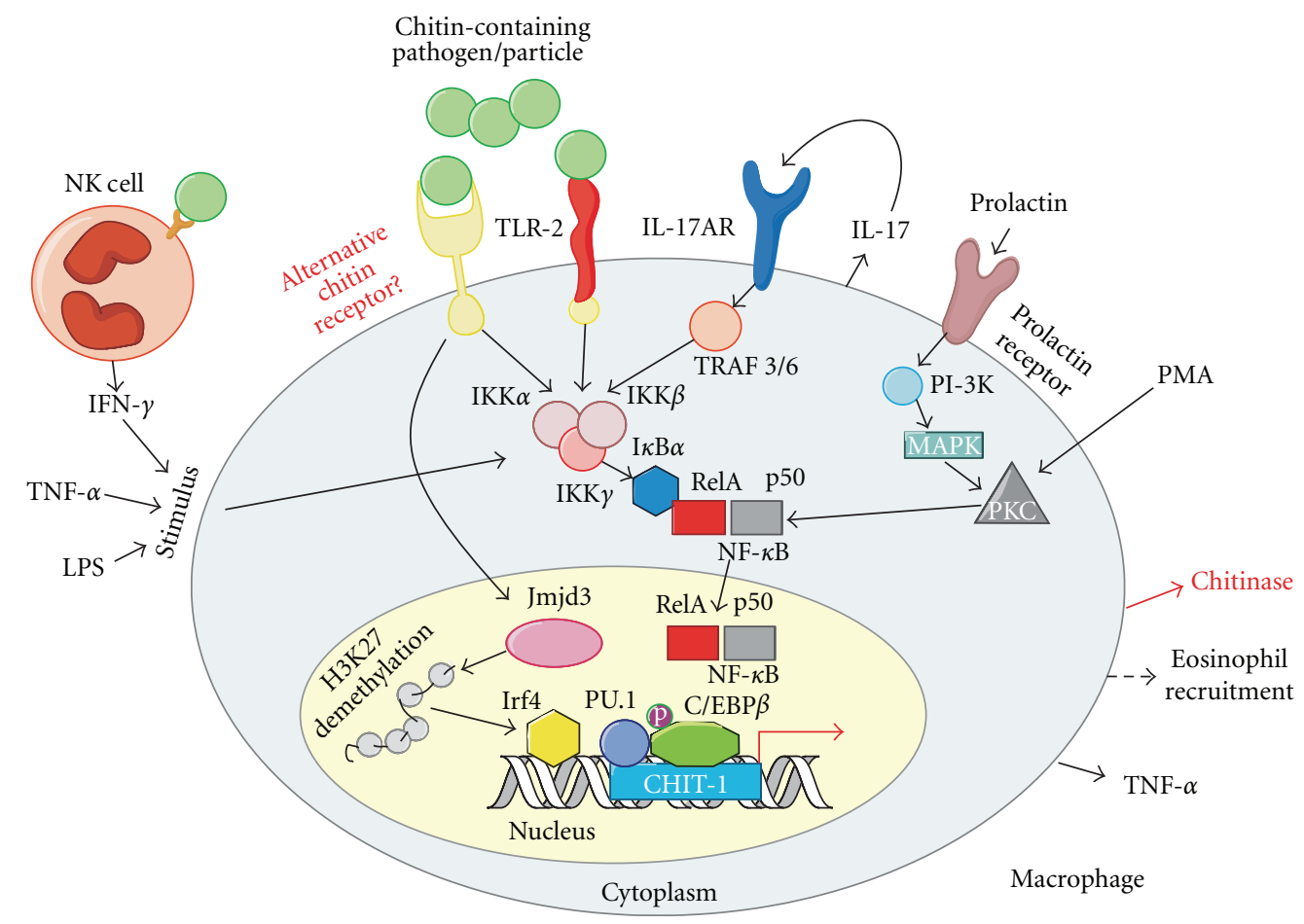

FIgURe 3: Hypothetical model for a molecular chitinase response to chitin-containing pathogens or particles. Chitin recognition leads to the expression of chitinase, TNF- $\alpha$, IL-17, and eosinophil recruitment $[83,84]$. NF- $\kappa$ B serves as the primary signaling pathway involved in the recognition of most fungal cell wall components (see Figure 2) [45]; therefore, its involvement in the expression of chitinase in response to a chitin-containing pathogen or particle is highly likely. Secretion of IFN- $\gamma$ by NK cells induces macrophage priming caused by stimulation with a chitin-containing pathogen or particle [60-63]. IFN- $\gamma$, LPS, TNF- $\alpha$, and PMA up-regulate chitinase activity [16-19], possibly through an NF- $\kappa$ B inflammatory-mediated pathway. IL-17 secretion upon macrophage stimulation with chitin increased levels of IL-17-AR and was TLR-2 dependent [84]. Either TLR-2 or an alternative chitin-specific receptor may recognize chitin-containing pathogens or particles and mediate chitinase activity. Prolactin stimulation of macrophages leads to chitinase expression via a PI-3K, MAPK, and NF- $\kappa$ B pathway [16-19]. Activation of Jmjd3, leads to demethylation of H3K27 and recruitment of the transcription factor Irf4, which is associated with M2 macrophage polarization in response to chitin stimulation [59]. Expression of the chitinase encoded by the CHIT-1 gene is regulated via PU.1 and C/EBP $\beta$. The latter is phosphorylated (p) to induce CHIT-1 expression [85]. NF- $\kappa \mathrm{B}$, nuclear factor kappa-lightchain-enhancer of activated B cells; IFN- $\gamma$ : interferon gamma; NK cells: natural killer cells; LPS: lipopolysaccharide; TNF- $\alpha$ : tumor necrosis factor alpha; PMA: phorbol 12-myristate 13-acetate; IL-17: interleukin-17; IL-17-AR: interleukin-17A receptor; TLR-2: toll-like receptor-2; PI-3K: phosphatidylinositol 3-kinase; MAPK: Mitogen-activated protein kinase; Jmjd3: Jumonji domain containing-3; H3K27: histone 3 Lys27 demethylase; CHIT-1: chitotriosidase; C/EBP $\beta$ : CCAAT-enhancer-binding protein beta.

This mutant protein can bind chitin particles, but cannot degrade chitin $[20,86]$. Macrophages from individuals with this 24-bp duplication in the CHIT-1 gene produced CHIT1 RNA and small amounts of a $47 \mathrm{kDa}$ protein, but no enzymatically active chitotriosidase [20]. Approximately 30 $40 \%$ of the human population is heterozygous and 3-6\% is homozygous for this duplication $[20,77,86]$. The use of chitinase activity as a disease biomarker may therefore be limited to patients with at least one wild-type CHIT-1 allele.

The effect of environmental conditions on the occurrence of the most prominent chitinase polymorphism, the 24bp duplication, was studied by Malaguarnera et al. DNA analysis was performed to compare the frequency of the exon 10 duplication allele in individuals from Mediterranean countries and sub-Saharan regions. This study found a higher frequency of individuals homozygous for the 24bp duplication in Sicily and Sardinia, 3.73\% and 5.45\%, respectively, than in people from Benin and Burkina
Faso (frequency of $0 \%$ homozygous for the duplication). The authors concluded that the presence of the CHIT-1inactivating 24-bp duplication in Sicily and Sardinia was due to the improved, more sanitary environmental conditions as compared to Benin and Burkina Faso, which still face widespread parasitic diseases and the presence of multiple chitin-containing pathogens [77]. The lack of chitotriosidase activity in people with these polymorphisms may be compensated for by AMCase chitinase activity. However, there are also various polymorphisms that affect AMCase activity $[22,23,87]$. Therefore, a thorough immunogenetic haplotype analysis that involves CHIT-1 and AMCase alleles, as well as chitin sensing and chitinase regulation pathways is needed to investigate the significance of human chitinase responses to fungal infections. It is possible that the dysregulation of chitin sensing or chitinase induction pathways could be associated with altered susceptibility for IFIs. 


\section{Concluding Remarks and Future Directions}

An efficient method for early diagnosis and treatment of IFIs is needed [27, 30]. Exploiting host responses to IFIs could help to better understand fungal recognition by the immune system, and may reveal potential diagnostic markers of IFIs. A substantial increase in chitinase activity, in conjunction with other IFIs clinical signs and symptoms, or in conjunction with the $\beta$-1,3-glucan assay could be a biomarker indicative of a beginning fungal infection. Chitinase activity appears to play an important role in various diseases $[13,68]$, and therefore, a clear understanding of the processes of chitinase induction and regulation is desirable.

Chitinases can be induced by various stimuli including prolactin, TNF- $\alpha$, IFN- $\gamma$, and PMA. And recombinant human CHIT-1 has demonstrated antifungal properties both in vivo and in vitro $[25,67]$. Therefore, it is conceivable that artificial induction of chitinase production in patients that are at risk of fungal infections could increase their resistance to fungal pathogens. This strategy would be most effective in patients with genes encoding catalytically active chitinases. In summary, chitinase-based diagnostic assay or antifungal therapeutics may be developed in the near future.

\section{Acknowledgments}

The authors are grateful to Dr. Keely Walker (City of Hope) for assistance with editing this paper. K. Vega and M. Kalkum are supported by the Hermann Foundation and the Tim Nesvig Lymphoma Fellowship and Research Fund.

\section{References}

[1] N. G. Almyroudis and B. H. Segal, "Antifungal prophylaxis and therapy in patients with hematological malignancies and hematopoietic stem cell transplant recipients," Expert Review of Anti-Infective Therapy, vol. 8, no. 12, pp. 1451-1466, 2010.

[2] H. L. Leather and J. R. Wingard, "New strategies of antifungal therapy in hematopoietic stem cell transplant recipients and patients with hematological malignancies," Blood Reviews, vol. 20, no. 5, pp. 267-287, 2006.

[3] L. Romani, "Immunity to fungal infections," Nature Reviews Immunology, vol. 4, no. 1, pp. 11-23, 2004.

[4] M. Li, Z. H. Liu, Q. Chen et al., "Insoluble $\beta$-glucan from the cell wall of Candida albicans induces immune responses of human THP-1 monocytes through Dectin-1," Chinese Medical Journal, vol. 122, no. 5, pp. 496-501, 2009.

[5] G. D. Brown, "Innate antifungal immunity: the key role of phagocytes," Annual Review Immunology, vol. 29, pp. 1-21, 2011.

[6] P. Kankkunen, L. Teirilä, J. Rintahaka, H. Alenius, H. Wolff, and S. Matikainen, "( 1,3$)$ - $\beta$-glucans activate both dectin-1 and NLRP3 inflammasome in human macrophages," Journal of Immunology, vol. 184, no. 11, pp. 6335-6342, 2010.

[7] J. P. Latge, "The cell wall: a carbohydrate armour for the fungal cell,” Molecular Microbiology, vol. 66, no. 2, pp. 279-290, 2007.

[8] H. M. Cauchie, "Chitin production by arthropods in the hydrosphere," Hydrobiologia, vol. 470, pp. 63-96, 2002.

[9] R. Garrett and C. M. Grisham, "Carbohydrates and the glycoconjugates of cell surfaces," in Biochemistry, chapter 7, p. 181, Cengage Learning, Boston, Mass, USA, 4th edition, 2010.
[10] G. H. Renkema, R. G. Boot, F. L. Au et al., "Chitotriosidase a chitinase, and the 39-kDa human cartilage glycoprotein, a chitin-binding lectin, are homologues of family 18 glycosyl hydrolases secreted by human macrophages," European Journal of Biochemistry, vol. 251, no. 1-2, pp. 504-509, 1998.

[11] R. G. Boot, A. P. Bussink, M. Verhoek, P. A. J. De Boer, A. F. M. Moorman, and J. M. F. G. Aerts, "Marked differences in tissuespecific expression of chitinases in mouse and man," Journal of Histochemistry and Cytochemistry, vol. 53, no. 10, pp. 12831292, 2005.

[12] R. G. Boot, E. F. C. Blommaart, E. Swart et al., "Identification of a novel acidic mammalian chitinase distinct from chitotriosidase," Journal of Biological Chemistry, vol. 276, no. 9, pp. 6770-6778, 2001.

[13] J. Kzhyshkowska, A. Gratchev, and S. Goerdt, "Human chitinases and chitinase-like proteins as indicators for inflammation and cancer," Biomark Insights, vol. 2, pp. 128-146, 2007.

[14] A. P. Bussink, D. Speijer, J. M. F. G. Aerts, and R. G. Boot, "Evolution of mammalian chitinase(-like) members of family 18 glycosyl hydrolases," Genetics, vol. 177, no. 2, pp. 959-970, 2007.

[15] B. Overdijk, G. J. Van Steijn, and F. C. Odds, "Chitinase levels in guinea pig blood are increased after systemic infection with Aspergillus fumigatus," Glycobiology, vol. 6, no. 6, pp. 627634, 1996.

[16] L. Malaguarnera, M. Musumeci, M. Di Rosa, A. Scuto, and S. Musumeci, "Interferon-gamma, tumor necrosis factor-alpha, and lipopolysaccharide promote chitotriosidase gene expression in human macrophages," Journal of Clinical Laboratory Analysis, vol. 19, no. 3, pp. 128-132, 2005.

[17] L. Malaguarnera, M. Musumeci, F. Licata, M. Di Rosa, A. Messina, and S. Musumeci, "Prolactin induces chitotriosidase gene expression in human monocyte-derived macrophages," Immunology Letters, vol. 94, no. 1-2, pp. 57-63, 2004.

[18] M. Di Rosa, A. M. Zambito, A. R. Marsullo, G. Li Volti, and L. Malaguarnera, "Prolactin induces chitotriosidase expression in human macrophages through PTK, PI3-K, and MAPK pathways," Journal of Cellular Biochemistry, vol. 107, no. 5, pp. 881-889, 2009.

[19] M. Di Rosa, M. Musumeci, A. Scuto, S. Musumeci, and L. Malaguarnera, "Effect of interferon- $\gamma$, interleukin-10, lipopolysaccharide and tumor necrosis factor- $\alpha$ on chitotriosidase synthesis in human macrophages," Clinical Chemistry and Laboratory Medicine, vol. 43, no. 5, pp. 499-502, 2005.

[20] R. G. Boot, G. H. Renkema, M. Verhock et al., "The human chitotriosidase gene. Nature of inherited enzyme deficiency," Journal of Biological Chemistry, vol. 273, no. 40, pp. 2568025685, 1998.

[21] E. H. Choi, P. A. Zimmerman, C. B. Foster et al., "Genetic polymorphisms in molecules of innate immunity and susceptibility to infection with Wuchereria bancrofti in South India," Genes and Immunity, vol. 2, no. 5, pp. 248-253, 2001.

[22] S. Bierbaum, A. Superti-Furga, and A. Heinzmann, "Genetic polymorphisms of chitotriosidase in Caucasian children with bronchial asthma," International Journal of Immunogenetics, vol. 33, no. 3, pp. 201-204, 2006.

[23] S. Bierbaum, R. Nickel, A. Koch et al., "Polymorphisms and haplotypes of acid mammalian chitinase are associated with bronchial asthma," The American Journal of Respiratory and Critical Care Medicine, vol. 172, no. 12, pp. 1505-1509, 2005. 
[24] A. P. Bussink, M. Verhoek, J. Vreede et al., "Common G102S polymorphism in chitotriosidase differentially affects activity towards 4-methylumbelliferyl substrates," FEBS Journal, vol. 276, no. 19, pp. 5678-5688, 2009.

[25] C. Gordon-Thomson, A. Kumari, L. Tomkins et al., "Chitotriosidase and gene therapy for fungal infections," Cellular and Molecular Life Sciences, vol. 66, no. 6, pp. 1116-1125, 2009.

[26] S. J. Park and B. Mehrad, "Innate immunity to Aspergillus species," Clinical Microbiology Reviews, vol. 22, no. 4, pp. 535$551,2009$.

[27] C. Garcia-Vidal, A. Upton, K. A. Kirby, and K. A. Marr, "Epidemiology of invasive mold infections in allogeneic stem cell transplant recipients: biological risk factors for infection according to time after transplantation," Clinical Infectious Diseases, vol. 47, no. 8, pp. 1041-1050, 2008.

[28] K. A. Marr, R. A. Carter, M. Boeckh, P. Martin, and L. Corey, "Invasive aspergillosis in allogeneic stem cell transplant recipients: changes in epidemiology and risk factors," Blood, vol. 100, no. 13, pp. 4358-4366, 2002.

[29] G. Morace and E. Borghi, "Fungal infections in ICU patients: epidemiology and the role of diagnostics," Minerva Anestesiologica, vol. 76, no. 11, pp. 950-956, 2010.

[30] J. P. Latge, "Aspergillus fumigatus and aspergillosis," Clinical Microbiology Reviews, vol. 12, no. 2, pp. 310-350, 1999.

[31] H. Liu, "Co-regulation of pathogenesis with dimorphism and phenotypic switching in Candida albicans, a commensal and a pathogen," International Journal of Medical Microbiology, vol. 292, no. 5-6, pp. 299-311, 2002.

[32] F. C. Odds, "Molecular phylogenetics and epidemiology of candida albicans," Future Microbiology, vol. 5, no. 1, pp. 6779, 2010.

[33] J. Zirkel, H. Klinker, A. Kuhn et al., "Epidemiology of Candida blood stream infections in patients with hematological malignancies or solid tumors," Medical Mycology. In press.

[34] M. Giacchino, N. Chiapello, S. Bezzio et al., "Aspergillus galactomannan enzyme-linked immunosorbent assay crossreactivity caused by invasive Geotrichum capitatum," Journal of Clinical Microbiology, vol. 44, no. 9, pp. 3432-3434, 2006.

[35] R. R. Klont, M. A. S. H. Mennink-Kersten, and P. E. Verweij, "Utility of Aspergillus antigen detection in specimens other than serum specimens," Clinical Infectious Diseases, vol. 39, no. 10, pp. 1467-1474, 2004.

[36] M. Weisser, C. Rausch, A. Droll et al., "Galactomannan does not precede major signs on a pulmonary computerized tomographic scan suggestive of invasive aspergillosis in patients with hematological malignancies," Clinical Infectious Diseases, vol. 41, no. 8, pp. 1143-1149, 2005.

[37] A. Jathavedam, D. C. Duré, Y. Taur, and D. M. Weinstock, "Limited utility of serum galactomannan assay after autoSCT," Bone Marrow Transplantation, vol. 44, no. 1, pp. 59-61, 2009.

[38] T. Miyazaki, S. Kohno, K. Mitsutake et al., "Plasma ( $1 \rightarrow 3)-\beta$ $\mathrm{D}$-glucan and fungal antigenemia in patients with candidemia, aspergillosis, and cryptococcosis," Journal of Clinical Microbiology, vol. 33, no. 12, pp. 3115-3118, 1995.

[39] L. Senn, J. O. Robinson, S. Schmidt et al., "1,3- $\beta$-D-glucan antigenemia for early diagnosis of invasive fungal infections in neutropenic patients with acute leukemia," Clinical Infectious Diseases, vol. 46, no. 6, pp. 878-885, 2008.

[40] A. Kedzierska, " $(1 \rightarrow 3)-\beta$-D-glucan-a new marker for the early serodiagnosis of deep-seated fungal infections in humans," Polish Journal of Microbiology, vol. 56, no. 1, pp. 3-9, 2007.
[41] Z. Racil, I. Kocmanová, B. Weinbergerová et al., "Detection of 1,3-beta-D glucan for diagnosis of invasive fungal infections in hematooncological patients: usefulness for screening of invasive mycosis and for confirmation of galactomannan positive results," Klinická Mikrobiologie a Infenkcní lékarství, vol. 15, no. 2, pp. 48-57, 2009.

[42] I. Kocmanova, Z. Rácil, D. Koukalová, and J. Mayer, "1,3-BetaD-glucan measurement and its usefulness in the diagnosis of invasive fungal infections," Klinická Mikrobiologie a Infenkcní lékarství, vol. 14, no. 3, pp. 88-92, 2008.

[43] G. D. Brown, "Dectin-1 : a signalling non-TLR patternrecognition receptor," Nature Reviews Immunology, vol. 6, no. 1, pp. 33-43, 2006.

[44] M. G. Netea, G. D. Brown, B. J. Kullberg, and N. A. R. Gow, "An integrated model of the recognition of Candida albicans by the innate immune system," Nature Reviews Microbiology, vol. 6, no. 1, pp. 67-78, 2008.

[45] M. G. Netea, N. A. R. Gow, C. A. Munro et al., "Immune sensing of Candida albicans requires cooperative recognition of mannans and glucans by lectin and Toll-like receptors," Journal of Clinical Investigation, vol. 116, no. 6, pp. 1642-1650, 2006.

[46] E. P. McGreal, M. Rosas, G. D. Brown et al., "The carbohydrate-recognition domain of Dectin-2 is a C-type lectin with specificity for high mannose," Glycobiology, vol. 16, no. 5, pp. 422-430, 2006.

[47] K. Sato, X. L. Yang, T. Yudate et al., "Dectin-2 is a pattern recognition receptor for fungi that couples with the $\mathrm{Fc}$ receptor $\gamma$ chain to induce innate immune responses," Journal of Biological Chemistry, vol. 281, no. 50, pp. 38854-38866, 2006.

[48] T. Jouault, M. El Abed-El Behi, M. Martínez-Esparza et al., "Specific recognition of Candida albicans by macrophages requires galectin-3 to discriminate Saccharomyces cerevisiae and needs association with TLR2 for signaling," Journal of Immunology, vol. 177, no. 7, pp. 4679-4687, 2006.

[49] C. Fradin, D. Poulain, and T. Jouault, " $\beta-1,2$-linked oligomannosides from Candida albicans bind to a 32-kilodalton macrophage membrane protein homologous to the mammalian lectin galectin-3," Infection and Immunity, vol. 68, no. 8, pp. 4391-4398, 2000.

[50] L. Kohatsu, D. K. Hsu, A. G. Jegalian, F. T. Liu, and L. G. Baum, "Galectin-3 induces death of Candida species expressing specific $\beta$-1,2-linked mannans," Journal of Immunology, vol. 177, no. 7, pp. 4718-4726, 2006.

[51] M. Yadav and J. S. Schorey, "The $\beta$-glucan receptor dectin1 functions together with TLR2 to mediate macrophage activation by mycobacteria," Blood, vol. 108, no. 9, pp. 31683175, 2006.

[52] H. S. Goodridge and D. M. Underhill, "Fungal recognition by TLR2 and Dectin-1," Handbook of Experimental Pharmacology, no. 183, pp. 87-109, 2008.

[53] T. Jouault, S. Ibata-Ombetta, O. Takeuchi et al., "Candida albicans phospholipomannan is sensed through toll-like receptors," Journal of Infectious Diseases, vol. 188, no. 1, pp. 165-172, 2003.

[54] M. Li, Q. Chen, Y. Shen, and W. Liu, "Candida albicans phospholipomannan triggers inflammatory responses of human keratinocytes through Toll-like receptor 2," Experimental Dermatology, vol. 18, no. 7, pp. 603-610, 2009.

[55] P. R. Taylor, S. V. Tsoni, J. A. Willment et al., "Dectin-1 is required for $\beta$-glucan recognition and control of fungal infection," Nature Immunology, vol. 8, no. 1, pp. 31-38, 2007. 
[56] B. P. Thornton, V. Větvička, M. Pitman, R. C. Goldman, and G. D. Ross, "Analysis of the sugar specificity and molecular location of the $\beta$-glucan-binding lectin site of complement receptor type 3 (CD11D/CD18)," Journal of Immunology, vol. 156, no. 3, pp. 1235-1246, 1996.

[57] V. Balloy and M. Chignard, "The innate immune response to Aspergillus fumigatus," Microbes and Infection, vol. 11, no. 12, pp. 919-927, 2009.

[58] P. V. Kasperkovitz, M. L. Cardenas, and J. M. Vyas, "TLR9 is actively recruited to Aspergillus fumigatus phagosomes and requires the $\mathrm{N}$-terminal proteolytic cleavage domain for proper intracellular trafficking," Journal of Immunology, vol. 185, no. 12, pp. 7614-7622, 2010.

[59] T. Satoh, O. Takeuchi, A. Vandenbon et al., "The Jmjd3-Irf4 axis regulates M2 macrophage polarization and host responses against helminth infection," Nature Immunology, vol. 11, no. 10, pp. 936-944, 2010.

[60] Y. Shibata, W. James Metzger, and Q. N. Myrvik, "Chitin particle-induced cell-mediated immunity is inhibited by soluble mannan: mannose receptor-mediated phagocytosis initiates IL-12 production," Journal of Immunology, vol. 159, no. 5, pp. 2462-2467, 1997.

[61] Y. Shibata, L. A. Foster, W. J. Metzger, and Q. N. Myrvik, "Alveolar macrophage priming by intravenous administration of chitin particles, polymers of N-acetyl-D-glucosamine, in mice," Infection and Immunity, vol. 65, no. 5, pp. 1734-1741, 1997.

[62] Y. Shibata, L. A. Foster, J. F. Bradfield, and Q. N. Myrvik, "Oral administration of chitin down-regulates serum IgE levels and lung eosinophilia in the allergic mouse," Journal of Immunology, vol. 164, no. 3, pp. 1314-1321, 2000.

[63] Y. Shibata, I. Honda, J. P. Justice, M. R. Van Scott, R. M. Nakamura, and Q. N. Myrvik, "Th1 adjuvant N-acetyl-Dglucosamine polymer up-regulates Th1 immunity but downregulates Th2 immunity against a mycobacterial protein (MPB-59) in interleukin-10-knockout and wild-type mice," Infection and Immunity, vol. 69, no. 10, pp. 6123-6130, 2001.

[64] C. Ozdemir, D. Yazi, M. Aydogan et al., "Treatment with chitin microparticles is protective against lung histopathology in a murine asthma model," Clinical and Experimental Allergy, vol. 36, no. 7, pp. 960-968, 2006.

[65] R. A. A. Muzzarelli, "Chitins and chitosans as immunoadjuvants and non-allergenic drug carriers," Marine Drugs, vol. 8, no. 2, pp. 292-312, 2010.

[66] P. Strong, H. Clark, and K. Reid, "Intranasal application of chitin microparticles down-regulates symptoms of allergic hypersensitivity to Dermatophagoides pteronyssinus and Aspergillus fumigatus in murine models of allergy," Clinical and Experimental Allergy, vol. 32, no. 12, pp. 1794-1800, 2002.

[67] M. van Eijk, C. P. A. A. van Roomen, G. H. Renkema et al., "Characterization of human phagocyte-derived chitotriosidase, a component of innate immunity," International Immunology, vol. 17, no. 11, pp. 1505-1512, 2005.

[68] A. P. Bussink, M. van Eijk, G. H. Renkema, J. M. Aerts, and R. G. Boot, "The biology of the Gaucher cell: the cradle of human chitinases," International Review of Cytology, vol. 252, pp. 71128, 2006.

[69] J. M. Aerts, M. J. van Breemen, A. P. Bussink et al., "Biomarkers for lysosomal storage disorders: identification and application as exemplified by chitotriosidase in Gaucher disease," Acta Paediatrica, International Journal of Paediatrics, vol. 97, no. 457, pp. 7-14, 2008.
[70] P. Alfonso, A. Cenarro, J. I. Pérez-Calvo et al., "Effect of enzyme replacement therapy on lipid profile in patients with Gaucher's disease," Medicina Clinica, vol. 120, no. 17, pp. 641646, 2003.

[71] P. Giraldo, A. Cenarro, P. Alfonso et al., "Chitotriosidase genotype and plasma activity in patients with type 1 gaucher's disease and their relatives (carriers and noncarriers)," Haematologica, vol. 86, no. 9, pp. 977-984, 2001.

[72] R. G. Boot, G. H. Renkema, A. Strijland, A. J. Van Zonneveld, and J. M. F. G. Aerts, "Cloning of a cDNA encoding chitotriosidase, a human chitinase produced by macrophages," Journal of Biological Chemistry, vol. 270, no. 44, pp. 2625226256, 1995.

[73] G. H. Renkema, R. G. Boot, A. O. Muijsers, W. E. DonkerKoopman, and J. M. F. G. Aerts, "Purification and characterization of human chitotriosidase, a novel member of the chitinase family of proteins," Journal of Biological Chemistry, vol. 270, no. 5, pp. 2198-2202, 1995.

[74] M. Ramanathan Jr., W. K. Lee, and A. P. Lane, "Increased expression of acidic mammalian chitinase in chronic rhinosinusitis with nasal polyps," The American Journal of Rhinology, vol. 20 , no. 3, pp. 330-335, 2006.

[75] F. Gianfrancesco and S. Musumeci, "The evolutionary conservation of the human chitotriosidase gene in rodents and primates," Cytogenetic and Genome Research, vol. 105, no. 1, pp. 54-56, 2004.

[76] J. Labadaridis, E. Dimitriou, C. Costalos et al., "Serial chitotriosidase activity estimations in neonatal systemic candidiasis," Acta Paediatrica, vol. 87, no. 5, p. 605, 1998.

[77] L. Malaguarnera, J. Simporè, D. A. Prodi et al., "A 24-bp duplication in exon 10 of human chitotriosidase gene from the sub-Saharan to the Mediterranean area: role of parasitic diseases and environmental conditions," Genes and Immunity, vol. 4, no. 8, pp. 570-574, 2003.

[78] M. G. Nair, K. J. Guild, and D. Artis, "Novel effector molecules in type 2 inflammation: lessons drawn from helminth infection and allergy," Journal of Immunology, vol. 177, no. 3, pp. 1393-1399, 2006.

[79] B. Overdijk, G. J. van Steijn, and F. C. Odds, "Distribution of chitinase in guinea pig tissues and increases in levels of this enzyme after systemic infection with Aspergillus fumigatus," Microbiology, vol. 145, no. 1, pp. 259-269, 1999.

[80] A. G. Vicencio, S. Narain, Z. Du et al., "Pulmonary cryptococcosis induces chitinase in the rat," Respiratory research, vol. 9, p. 40, 2008.

[81] T. A. Korolenko, S. Y. Zhanaeva, O. V. Falameeva et al., "Chitotriosidase as a marker of macrophage stimulation," Bulletin of Experimental Biology and Medicine, vol. 130, no. 10, pp. 948-950, 2000.

[82] C. Gorzelanny, B. Pöppelmann, K. Pappelbaum, B. M. Moerschbacher, and S. W. Schneider, "Human macrophage activation triggered by chitotriosidase-mediated chitin and chitosan degradation," Biomaterials, vol. 31, no. 33, pp. 85568563, 2010.

[83] T. A. Reese, H. E. Liang, A. M. Tager et al., "Chitin induces accumulation in tissue of innate immune cells associated with allergy," Nature, vol. 447, no. 7140, pp. 92-96, 2007.

[84] C. A. Da Silva, D. Hartl, W. Liu, C. G. Lee, and J. A. Elias, "TLR-2 and IL-17A in chitin-induced macrophage activation and acute inflammation," Journal of Immunology, vol. 181, no. 6, pp. 4279-4286, 2008.

[85] T. H. Pham, S. Langmann, L. Schwarzfischer et al., "CCAAT enhancer-binding protein $\beta$ regulates constitutive gene expression during late stages of monocyte to macrophage 
differentiation," Journal of Biological Chemistry, vol. 282, no. 30, pp. 21924-21933, 2007.

[86] M. R. Rodrigues, M. C. Sa Miranda, and O. Amaral, "Allelic frequency determination of the 24-bp chitotriosidase duplication in the Portuguese population by real-time PCR," Blood Cells, Molecules, and Diseases, vol. 33, no. 3, pp. 362-364, 2004.

[87] M. A. Seibold, T. A. Reese, S. Choudhry et al., "Differential enzymatic activity of common haplotypic versions of the human acidic mammalian chitinase protein," Journal of Biological Chemistry, vol. 284, no. 29, pp. 19650-19658, 2009. 

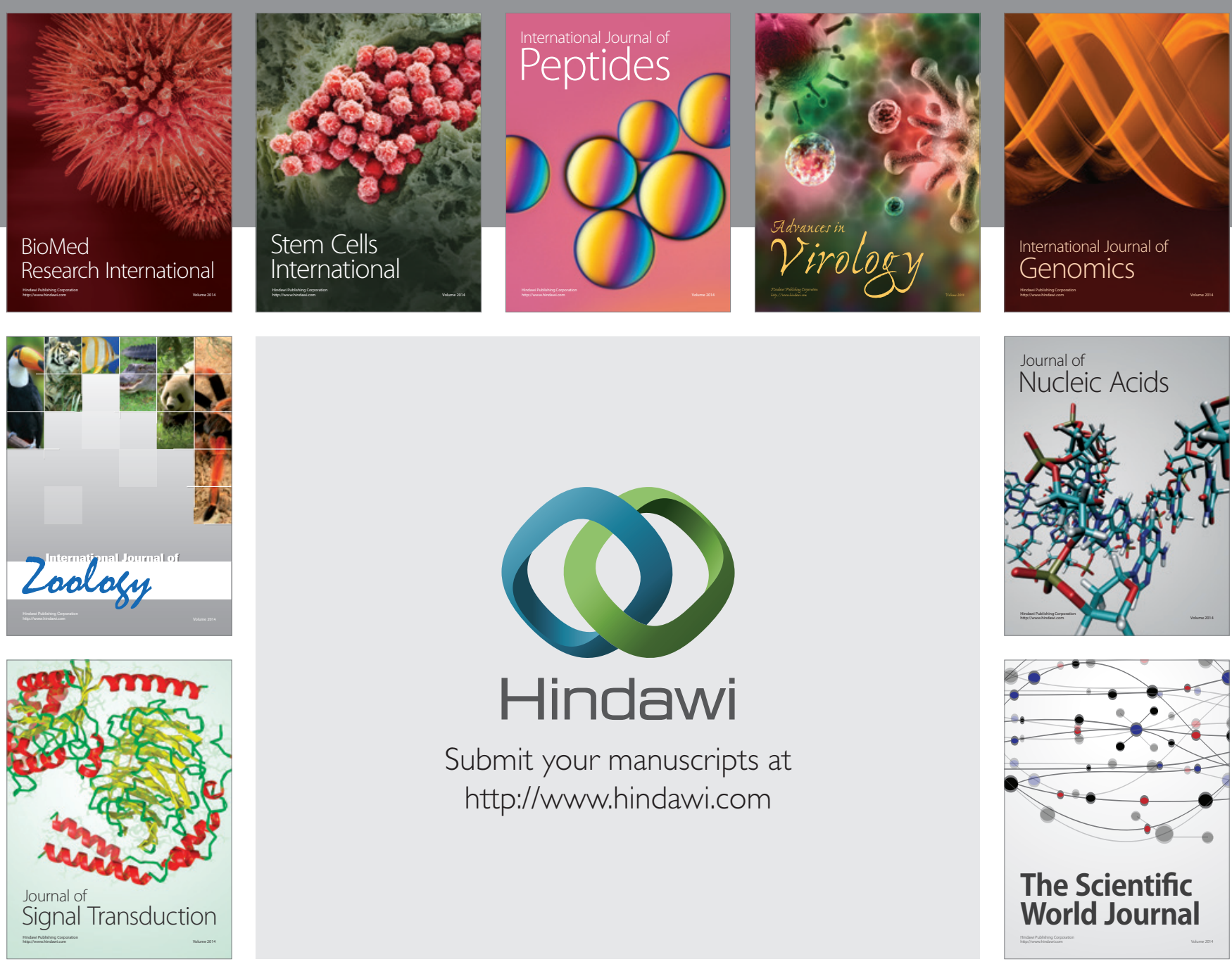

Submit your manuscripts at

http://www.hindawi.com
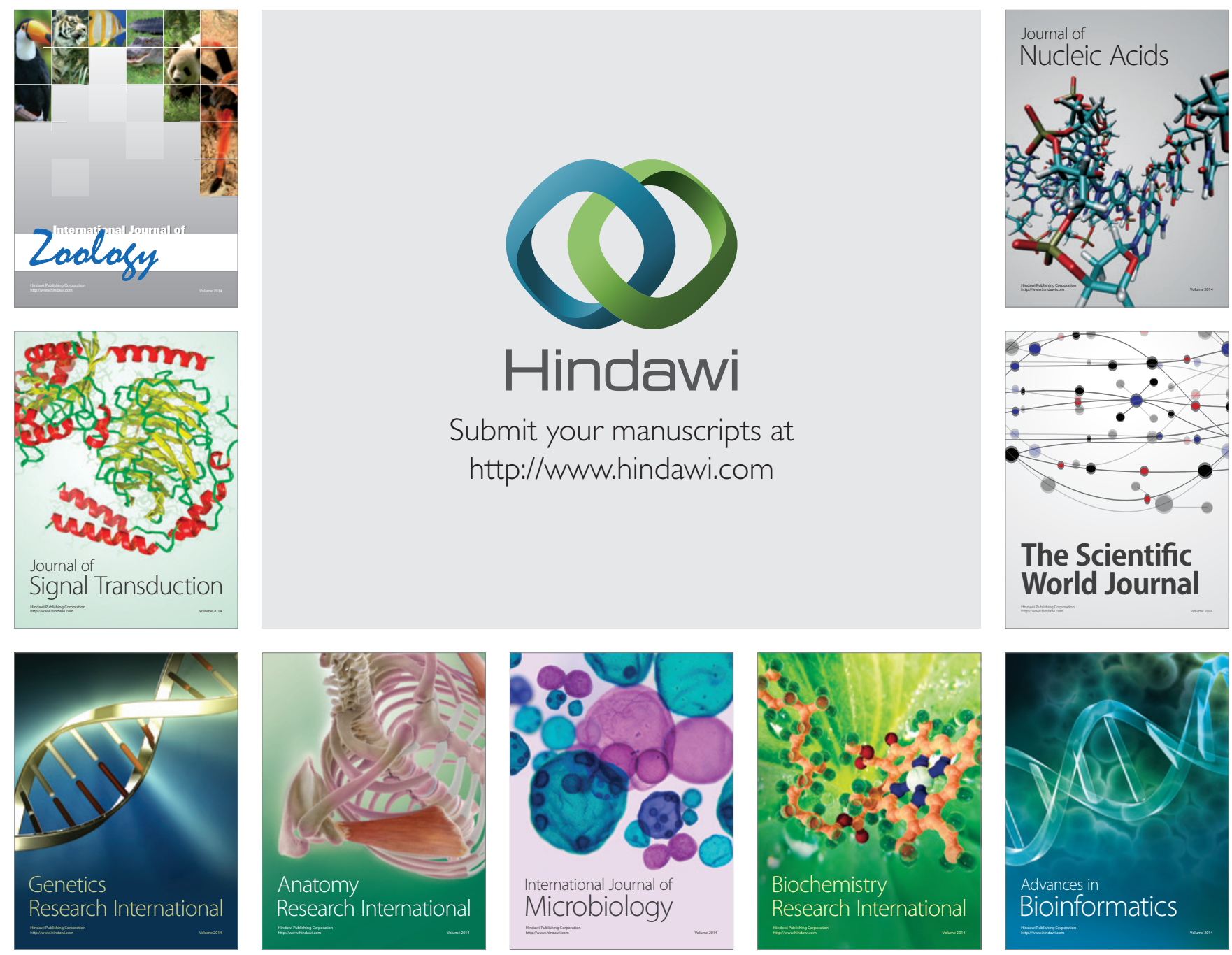

The Scientific World Journal
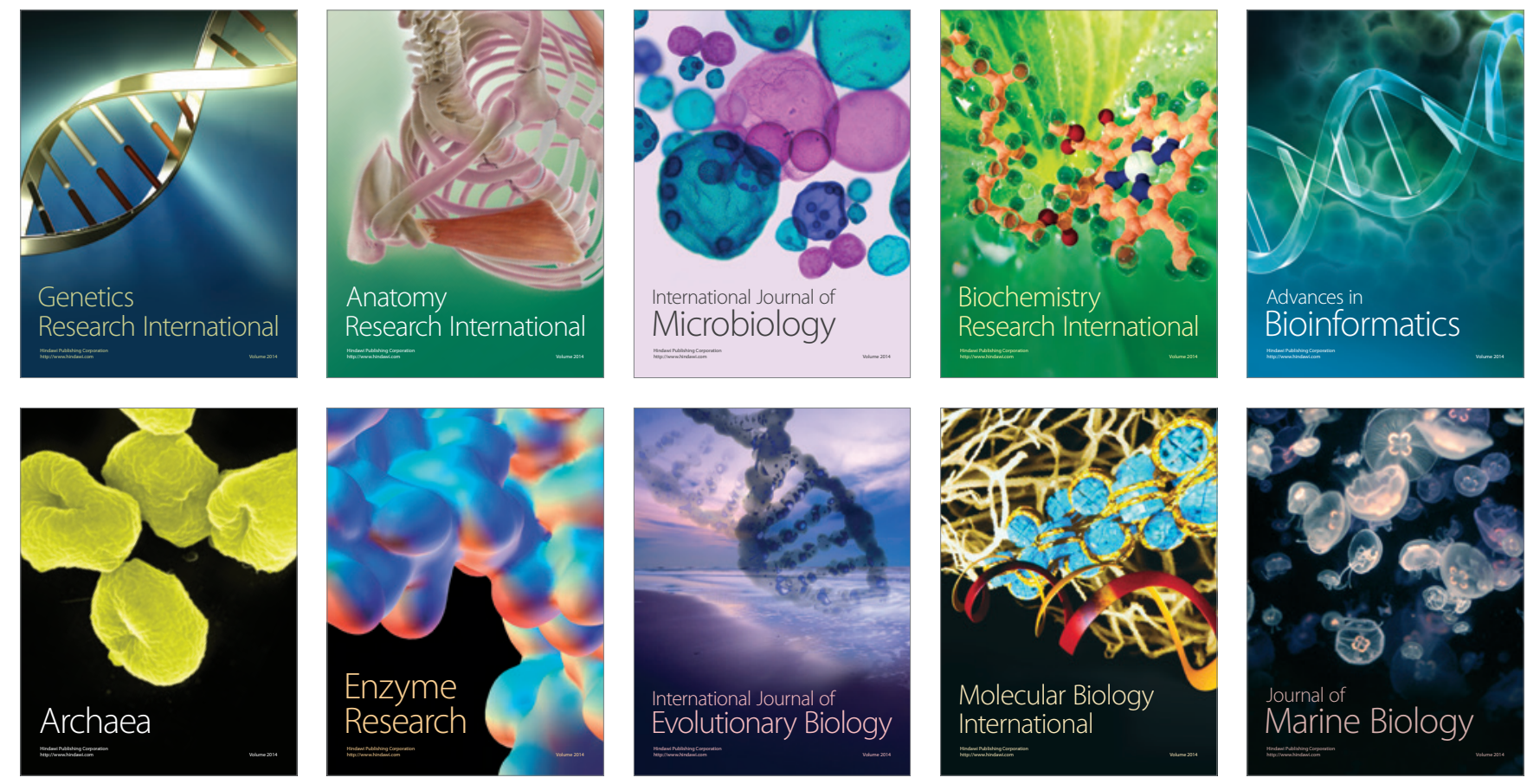